\title{
Teleprogramming Service Provides Safe and Remote Stimulation Options for Patients with DRG-S and SCS Implants
}

\author{
Timothy R Deer (D) \\ Michael F Esposito ${ }^{2}$ \\ Eric G Cornidez ${ }^{3}$ \\ Udoka Okaro (1D ${ }^{4}$ \\ Marie E Fahey ${ }^{4}$ \\ Kenneth B Chapman (iD ${ }^{5}$ \\ 'Spine and Nerve Center of the Virginias, \\ Charleston, WV, USA; ${ }^{2}$ Florida Pain \\ Institute, Melbourne, FL, USA; ${ }^{3}$ Pain \\ Institute of Southern Arizona, Tucson, \\ AZ, USA; ${ }^{4}$ Abbott, Austin, TX, USA; \\ ${ }^{5}$ The Spine Institute of New York, \\ New York, NY, USA
}

Correspondence: Marie E Fahey

Abbott, 870I Bee Cave Road, Bldg I

West FM 2244, Austin, TX, 78746, USA

Tel + I 7372089348

Fax + I 737-309-87I0

Email marie.fahey@abbott.com
Background: Chronic pain patients implanted with a neurostimulation device typically require follow-up and device programming visits to address changes in symptoms or treatment. Follow-up visits require access to specialty care and necessitate patients to take time off work, commute long distances, arrange for travel, and/or work with a caregiver's schedule. Telemedicine was adopted for some patient management as a result of the SarsCov-2 pandemic; however, remote optimization for neuromodulation still required an inperson visit to adjust device parameters. An FDA-approved digital platform enables remote programming of an implanted neuromodulation device using a real-time audio-video link from the clinical programmer to the patient controller. The Remote Optimization, Adjustment, and Measurement for Chronic Pain Therapy (ROAM-CPT) is a multi-center, prospective study that is currently underway to access the effectiveness of the teleprogramming system in fulfilling patients' clinical demands.

Methods: This pilot study surveyed 16 patients to determine the ability of the teleprogramming platform to provide a rapid solution safely and effectively for patient's chronic pain. Data were collected using a questionnaire that asked 6 clinician-centric questions and 5 patient-centric questions.

Results: 4/4 surveyed physicians were able to address patients' needs. 16/16 surveyed patients reported a quick resolution to pain and 15/16 did not require additional follow-up. Data curated from this pilot study show that the teleprogramming application greatly improves patient care, is preferred by both clinicians and patients with minimal disruptions to patients' everyday lives.

Conclusion: Teleprogramming provides real-time virtual programming capabilities and optimizes patients' therapy.

Perspective: This article describes remote device programming and analysis as an alternative to in-person programming/treatment sessions for neuromodulation patients. This remote option gives patients access to timely and clinically appropriate device management when in-person care may not be available.

Keywords: neuromodulation, neurosphere, remote DRG stimulation, remote SCS stimulation, virtual clinic

\section{Introduction}

About $20.4 \%$ of US adults (50 million) suffer from chronic pain with $8 \%$ of individuals suffering from high-impact chronic pain. ${ }^{1}$ Chronic pain has a significant economic impact, with annual costs estimated at $\$ 635$ billion. $^{2,3}$ It has been linked to loss of mobility, opioid dependence, anxiety, and depression, 
leading to an overall decrease in quality of life. ${ }^{1}$ Additionally, up to $15 \%$ of all chronic pain patients are unresponsive to standard therapy, and therefore, seek alternative options. ${ }^{2}$ Spinal cord stimulation (SCS) and dorsal root ganglion stimulation (DRG-S) are two FDA-approved neuromodulation therapies that safely address chronic pain. Both are minimally invasive, reversible procedures in which electrical currents are applied to neural structures via leads either placed in the patient's epidural space (SCS) stimulating the dorsal column fibers or on the dorsal root ganglion (DRG). Both SCS and DRG-S have been successfully used to treat a variety of neuropathic pain conditions including failed back surgery syndrome (FBBS), ${ }^{4,5}$ complex regional pain syndrome (CRPS), ${ }^{6-9}$ axial back pain, ${ }^{10}$ ischemic limb pain, ${ }^{11}$ refractory unilateral limb pain syndrome, ${ }^{12}$ phantom limb pain, ${ }^{13-15}$ postherpetic neuralgia, ${ }^{16}$ acute herpes zoster pain, and discogenic pain. ${ }^{13}$ SCS and DRG-S reduce pain, have fewer serious adverse side-effects when compared to pharmacological options (such as opiate addiction), ${ }^{17}$ and less financial burden over 5 years when compared to other chronic pain therapies such as physical therapy. ${ }^{18,19}$ Changes in physiology, pathology, or movement of electrodes relative to the target tissue can lead to changes in the patient's symptoms. These modifications necessitate reprogramming of the neuromodulation devices, allowing a clinician to tailor therapy to the needs of individual patients and adapt therapy as symptoms change. For patients with neuromodulation systems, in-person followup visits are used to address programming and symptom changes. This necessitates patients traveling to their clinician's office. The financial burdens, disruptions to a patient's life, and/or travel inconvenience pose a substantial barrier to pain therapy for long commutes.

Travel constraints imposed during the COVID-19 pandemic disrupted healthcare access to millions of chronic pain patients. ${ }^{20}$ Telemedicine, the remote diagnosis and treatment of patients using telecommunications technology, has been used since the late 1990s. ${ }^{21}$ It became increasingly popular in 2020 in response to the pandemic, making healthcare accessible to individuals using highspeed internet and smartphones. ${ }^{22}$ Telemedicine is safe, cost-effective, provides intended therapeutics, improved patient compliance, satisfaction, and quality of life. ${ }^{23,24}$ Remote programming for SCS was studied in China in 2019-2020, 68.8\% of patients involved in the study preferred remote programming. ${ }^{25}$ Given the discomfort that may occur during travel for patients with chronic pain, risk of exposure during the COVID-19 pandemic, and the need for rapid resolution of any emerging symptoms, ${ }^{26}$ it is beneficial to investigate alternate pain management options. An FDA-approved digital teleprogramming platform (NeuroSphere (TM) Virtual Clinic, Abbott, Austin, Texas) allows remote programming of neuromodulation devices using a real-time audio-video connection. The software has secure remote video chat connectivity and clinicians can manipulate and adjust device functionality and parameters remotely, perform systems checks, and provide session reports.

A Remote Optimization, Adjustment, and Measurement for Chronic Pain Therapy (ROAM-CPT) study is currently underway and intended to demonstrate the capability of this teleprogramming system to:

- Safely interrogate and program neuromodulation devices used for chronic pain

- Effectively address patients' clinical needs by making adjustments to treat chronic pain without the need for additional in-person sessions

- Provide a rapid solution for patient's needs by comparing time to usage of remote care with in-person appointment wait times

This work is to our knowledge, is the first published report on SCS and DRG-S remote programming for chronic pain patients in the US.

\section{Method}

\section{Pilot Study Participants}

This pilot study was conducted at four sites within the United States. Eligible patients with access to an iPhone compatible with the teleprogramming software were required to sign an informed consent before registering for the teleprogramming application. A questionnaire was designed by medical affairs physicians and scientists to confirm the ability of participating physicians to diagnose a medical condition in patients without access to clinically appropriate in-person diagnostic services in addition to the treatment of chronic pain being completed more quickly and effectively. Patients were eligible to participate if they:

- Have an implantable, compatible SCS or DRG device

- Provide consent for the use of the teleprogramming service 


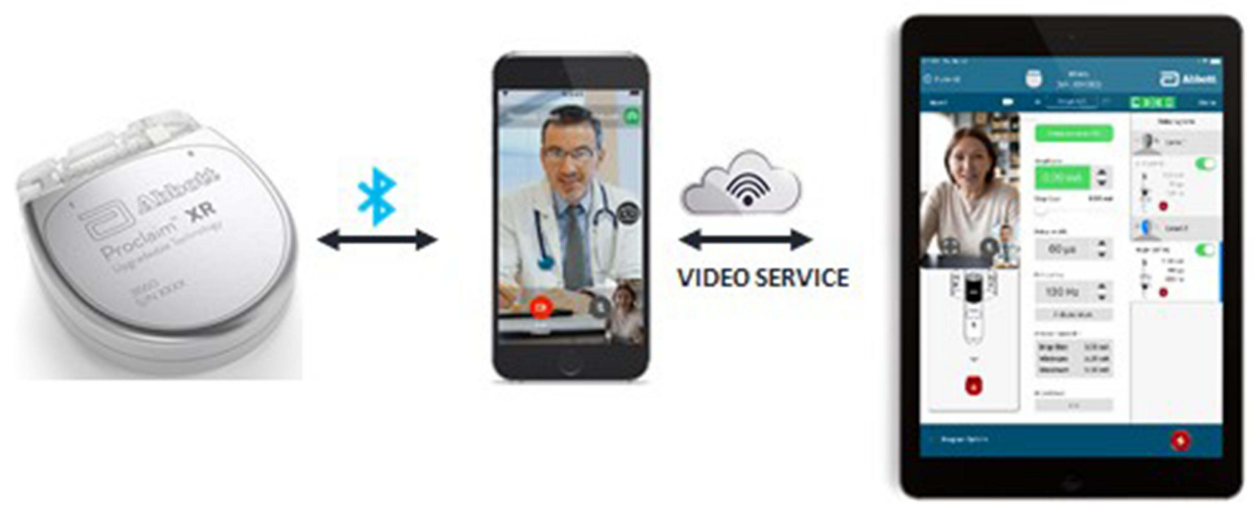

Figure I System used for remote care. Implanted neuromodulation device connects wirelessly to the iPhone patient controller using Bluetooth. The patient controller establishes an internet connection to the iPad clinician programmer with integrated audio and video. The clinician programmer has an inset video of the patient and access to the full set of programming controls.

- Are able to use the compatible, patient controller Apple iPhone

Refer to the ethical section below for consent information.

4 physicians (authors TD, ME, EC and $\mathrm{KC}$ ) and 16 patients implanted with SCS or DRG neuromodulation device participated in this pilot study. Data was collected using a questionnaire that asked 6 clinician-centric questions and 5 patient-centric questions.

\section{Initiating a Session}

An initial, one-time registration for access to the teleprogramming platform is required of the clinician and patient. Before establishing a remote connection, the patient controller must be within the Bluetooth range of the Implantable Pulse Generator (IPG) to maintain a connection and enable programming adjustments to the neurostimulator. The patient starts a video session using an internet connection. This generates a public key infrastructure (PKI) code which secures the session and enables the clinician to remotely connect to the patient's implantable generator. A security token is generated for each session granting the clinician access to perform therapy adjustments such as changing the frequency, pulse width, amplitude, and impedance during a therapeutic session (Figure 1). Sessions are initiated by patients as needed to address a therapeutic need. A session may be diagnostic (routine device check), simple programming (changes in 1-3 parameters), or a complex session (changes in more than 3 parameters). Table 1 summarizes the different types of remote sessions and applicable CPT reimbursement codes.

After each remote session, the patient and clinician were given an internally, designed questionnaire to complete. Clinician satisfaction with the teleprogramming platform was assessed using 6 criteria: (1) the ability to establish an audio-visual connection, (2) achieving the clinical goals of a programming session, (3) ability to complete a session, (4) reduction in the need for an in-person follow-up after a remote session, (5) providing rapid treatment resolution,

Table I Public Health Emergency (PHE) Code and Descriptors for Programming Sessions

\begin{tabular}{|c|c|c|c|c|c|c|}
\hline $\begin{array}{l}\text { CPT } \\
\text { Code }\end{array}$ & $\begin{array}{l}\text { Therapy } \\
\text { Area }\end{array}$ & Short Descriptor & $\begin{array}{l}\text { Work } \\
\text { RVU }\end{array}$ & Facility & $\begin{array}{l}\text { Non- } \\
\text { Facility }\end{array}$ & Status \\
\hline 95970 & $\begin{array}{l}\text { SCS/DRG/ } \\
\text { DBS }\end{array}$ & $\begin{array}{l}\text { Analysis without } \\
\text { programming }\end{array}$ & 0.35 & $\$ 19$ & $\$ 20$ & \multirow[t]{3}{*}{$\begin{array}{l}\text { Temporary addition for the PHE for the COVID-IS } \\
\text { Pandemic. Added I0//4/20 }\end{array}$} \\
\hline 95971 & SCS/DRG & $\begin{array}{l}\text { Analysis with simple } \\
\text { programming }\end{array}$ & 0.78 & $\$ 40$ & $\$ 50$ & \\
\hline 95972 & SCS/DRG & $\begin{array}{l}\text { Analysis with complex } \\
\text { programming }\end{array}$ & 0.80 & $\$ 42$ & $\$ 58$ & \\
\hline
\end{tabular}

Abbreviations: CPT, current procedural terminology; SCS, spinal cord stimulation; DRG, dorsal root ganglion; DBS, deep brain stimulation; RVU; relative value unit. 
and (6) preference for a remote vs an in-person session. Similarly, patient satisfaction was assessed using 5 criteria: satisfaction, preference, recommendation, resources saved, and the ability to complete a session.

\section{Results}

\section{Teleprogramming Provides Real-Time,} Safe Programming That Equals an

\section{in-Person Session}

All clinicians (4/4) who participated in the pilot study reported that they were able to establish an audio-video connection with all 16 subjects that participated and could provide the same service as an in-person session. Of the 16 established sessions, 8 sessions consisted of complex programming (3 or more parameters adjusted), 5 sessions were simple programming sessions (1-3 parameters adjusted), while the other 3 sessions were simple device interrogation (diagnostic check) without programming (Figure 2). All 4 clinicians reported that the 16 remote programming sessions led to rapid control of targeted chronic pain, and similarly 16/16 (100\%) of patients reported a quick resolution. All 16 patients required one session each to achieve the purpose of each session: Diagnostic or programming change. 15/16 (93.8\%) of patients who participated in this study did not require an in-person follow-up session to resolve their therapy needs and 1 patient required a follow-up visit for diagnostic imaging (X-ray) unrelated to using the teleprogramming session.

\section{Programming Session}

\section{- Complex Session \\ - Simple programming session \\ - Device interrogation/ Diagnostic check}

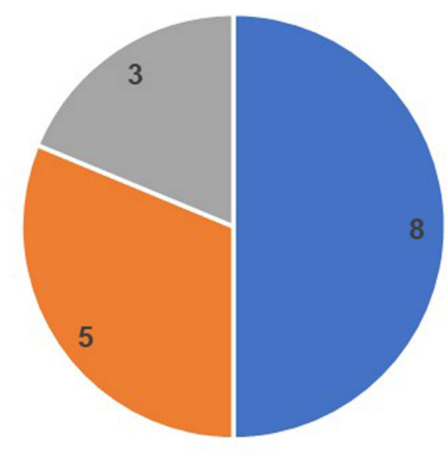

Figure 2 Outline of the pilot study programming session. 100\% of the participating physicians established audio-video connectivity and were able to provide a rapid, beneficial solution to patients' therapeutic needs. 8 complex programming sessions, 5 simple programming sessions and 3 diagnostic checks were conducted during the pilot study.

\section{Patient and Physician Satisfaction Rate}

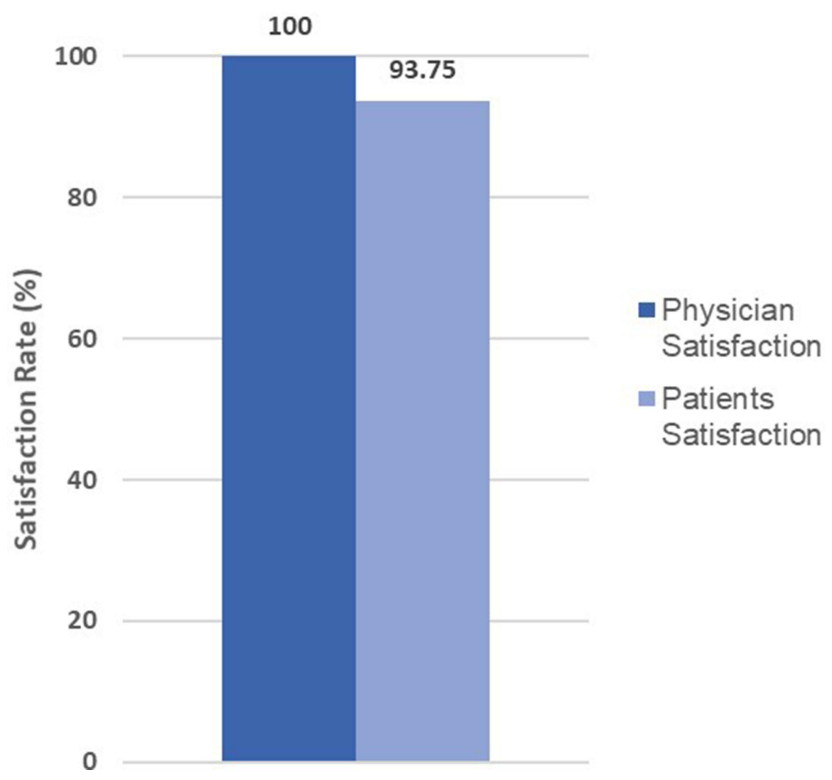

Figure 3 Physician and patients' satisfaction rate. $100 \%$ of physicians and $93.8 \%$ of patients would recommend the teleprogramming platform.

\section{Clinicians and Patients Prefer Teleprogramming}

All involved clinicians preferred remote programming to in-person sessions for future patient device checks, simple programming adjustments, or complex programming needs (Figure 3). 12/16 (75.0\%) of patients reported being able to get a remote appointment quickly, in comparison to scheduling an in-person appointment. 15/16 (93.8\%) of patients favored teleprogramming over inperson sessions for future programming adjustments (Figure 3). 6/16 (37.5\%) of patients stated before the availability of remote programming they had to commute 30mins-1 hour for an in-person appointment, $6 / 16$ (37.5\%) commute (1-2 hours), and 4/16 (25.0\%) had extensive travel time (more than 2 hours). 6/16 (37.5\%) had to arrange commute rides, travel with caregivers, arrange childcare, and/or arrange a hotel. Finally, 3/16 (18.75\%) reported having to take time off work for an in-person visit.

\section{Discussion}

Neuromodulation therapies like SCS and DRG are being used to treat a host of chronic pain conditions. While telemedicine has been used pre-pandemic, the COVID-19 pandemic primarily altered healthcare delivery by increasing the demand for telehealth. With COVID-19, there was 
a need to create applications that can enable clinicians to provide safe, uninterrupted access to patient care. Followup sessions also increase the financial burden of care and the burden of travel on patients who are experiencing increases in their symptoms. A previous internal survey for the test drive of the service showed that $74 \%$ of chronic pain therapy patients would prefer to have device adjustments without having to visit the clinic. Additionally, $48 \%$ of SCS/DRG chronic pain patients rely on a caretaker, friend, or family member to drive them to their appointments. Mendez et $\mathrm{al}^{27}$ studied the use of a remote-presence robot which required the presence of a healthcare provider for chronic pain programming. A few years later, Han et $\mathrm{al}^{25}$ studied remote programming on SCS patients in China with data indicating a preference for remote care.

Teleprogramming allows clinicians to make therapeutic program adjustments, optimizing patient care in real-time from different geographical locations. Clinicians maintain full functionality as an in-person session and can adjust patients' parameters like amplitude, polarity, pulse width, frequency, and add or remove programs to deliver optimum care.

Data from this pilot study shows that all clinicians were able to establish an audio-visual connection and completed either a device interrogation, a simple programming session, or a complex programming session with the same efficiency as an in-person session. All surveyed clinicians preferred the remote option to an in-person session (Figure 3) and only 1 patient required an un-related follow-up visit suggesting that the remote option achieves the same purpose as an in-person session without increasing the need for additional follow-up. To mitigate bias, surveyed clinicians had no input in survey design.

Of the 16 patients surveyed for this pilot study, 15/16 (93.8\%) preferred the remote option and were satisfied with the service (Figure 3), 1 patient showed no preference for an in-person vs a remote option. Most patients favored remote sessions because it was easier and faster to schedule an appointment hence providing a rapid and clinically beneficial solution for patients. Patients did not report any adverse effect from having IPG programmed virtually. Finally, all patients reported one or more of the following: saving time off work, saving extensive time (1-2 hours) required to commute to the appointment, not having to schedule caregivers, rides, and/or childcare, reducing the financial cost required to travel and travel accommodation where necessary.
The use of NeuroSphere ${ }^{\mathrm{TM}}$ was approved for temporary reimbursement codes (10/14/2020) under the Public Health Emergency (Table 1- CPT codes). CPT 95970 is reimbursed when a physician or other performs electronic analysis of a variety of parameters of a previously implanted neurostimulator pulse generator/transmitter to ensure functionality without reprogramming the device. In contrast, CPT codes 95971 and 95972 supports reimbursement for the electronic programming of an implanted neurostimulator pulse generator/transmitter (eg, changes in amplitude, pulse width, frequency [Hz], on/off cycling, burst, patient selectable parameters, responsive neurostimulation, detection algorithms, closed loop parameters, and passive parameters) by a physician or other qualified health care professional. CPT 95971 supports changing 13 parameters listed above and CPT 95972 supports changes in 3 or more parameters.

\section{Conclusion}

Teleprogramming connects a clinician and patient using a secure in-app video chat making it quicker for a clinician to optimize therapy with minimal disruption to the patient's life. Teleprogramming also provides real-time programming capabilities and optimizes patients' therapy. In summary, data from this pilot study shows that a teleprogramming option affords clinicians the ability to provide quick patient care, does not increase the need for additional follow-up, and in turn, reduces the burden of care placed on the patients while providing them clinical benefits. The ROAM-CPT study currently underway samples a larger population to ascertain clinical benefits from this pilot study.

\section{Ethics Statement}

The teleprogramming platform is already approved by the FDA. Data was collected using a questionnaire to access the safety and ability of the platform to provide quick patient care. By provision of consent, patient acknowledges that the use of the teleprogramming application is subject to a privacy notice and to the processing and transfer of personal information, including health-related information. Consent is granted at free will.

Patient Personal Information is collected for the following purposes: 1) to create Patient accounts, 2) to connect Patients and Clinicians and their associated Virtual Clinic Programmer and Controller Apps, 3) to allow Clinicians to contact Patients, 4) to allow teleprogramming representatives to contact Patients for support purposes, 
and 5) to allow Patients to access and use the platform to receive treatment.

Patient Personal Information collected and stored by the NeuroSphere ${ }^{\mathrm{TM}}$ includes:

- Personal data: first name, last name, email address, primary phone number, Clinician name

- Implantable Pulse Generator (IPG) model number, IPG Serial Number and manufacture date, whether the Patient Controller device is management company, serial number of managed mobile device, mapping of Patient device to Clinician account

- The patient's therapy type

- Information relating to the Patient's neurotherapy programs, program settings, IPG usage history, IP address and country.

Patients personal information is used to:

- Contact Clinical users about Virtual Clinic

- Learn about Virtual Clinic users and markets

- Assess and improve Virtual Clinic App performance

- Provide technical support and troubleshooting for the Virtual Clinic Apps

- Track and improve performance of Virtual Clinic Apps

- Develop internal organization reports for Virtual Clinic promotion, research, and Web portal and App content.

This survey did not require IRB consent according to CFR 45, PART 46-PROTECTION OF HUMAN SUBJECTS which states FDA requirement for exempt as "Research that only includes interactions involving educational tests (cognitive, diagnostic, aptitude, achievement), survey procedures, interview procedures, or observation of public behavior (including visual or auditory recording) if at least one of the following criteria is met: Any disclosure of the human subjects' responses outside the research would not reasonably place the subjects at risk of criminal or civil liability or be damaging to the subjects' financial standing, employability, educational advancement, or reputation".

The teleprogramming platform conforms to the Helsinki Declaration by accessing the platform for safety, effectiveness, efficiency, accessibility, and quality of care provided.

\section{Application and Device Status}

The Teleprogramming service and patients' implants are FDA-approved.

\section{Ethical Approval}

This pilot study was exempted from IRB approval due to removed patient identifiers, in accordance with US federal guidelines and regulations. Any disclosure of the human subjects' responses outside the research would not reasonably place the subjects at risk of criminal or civil liability or be damaging to the subjects' financial standing, employability, educational advancement, or reputation. This data was gathered from subjects who registered and consented for the FDA-approved teleprogramming platform. Consent is further defined in the Ethics declaration.

\section{Disclosure}

TRD consults for Axonics, Bioness, Nalu, Abbott, Spinethera, Flowonix, Saluda, Vertos, Vertiflex, and Cornerloc, has minor equity in Axonics, Spinethera, Saluda, Stimgenics, Medtronic, Nalu, Nevro, Vertiflex, and Vertos, and has conducted funded research for Vertiflex, Vertos, Abbott, Saluda, and Mainstay. TRD has a patent pending to Abbott. MFE is a consultant/advisor for Abbott, Boston Scientific, Flowonix, Nevro, and OrthoFundamentals. He conducts research with Abbott, Boston Scientific, Medtronic, and Nevro. EGC receives consulting fees from Abbott. He also conducts research for Abbott and Nevro. UO and MEF are employees of Abbott. The authors report no other conflicts of interest in this work.

\section{References}

1. Dahlhamer J, Lucas J, Zelaya C, et al. Prevalence of chronic pain and high-impact chronic pain among adults - United States, 2016. MMWR Morb Mortal Wkly Rep. 2018;67(36):1001-1006. doi:10.15585/mmwr. $\mathrm{mm} 6736 \mathrm{a} 2$

2. IOM (Institute of Medicine). Relieving Pain in America: A Blueprint for Transforming Prevention, Care, Education, and Research. Washington, DC: The National Academies Press; 2011.

3. Gaskin DJ, Richard P. The economic costs of pain in the United States. J Pain. 2012;13(8):715-724. doi:10.1016/j.jpain.2012.03.009

4. Kumar K, Taylor RS, Jacques L, et al. Spinal cord stimulation versus conventional medical management for neuropathic pain: a multicentre randomised controlled trial in patients with failed back surgery syndrome. Pain. 2007;132(1-2):179-188. doi:10.1016/j.pain.2007.07.028

5. Manchikanti L, Singh V, Pampati V, Smith HS, Hirsch JA. Analysis of growth of interventional techniques in managing chronic pain in the Medicare population: a 10-year evaluation from 1997 to 2006. Pain Physician. 2009;12(1):9-34. doi:10.36076/ppj.2009/12/9

6. Forouzanfar T, Kemler MA, Weber WE, Kessels AG, van Kleef M. Spinal cord stimulation in complex regional pain syndrome: cervical and lumbar devices are comparably effective. Br J Anaesth. 2004;92 (3):348-353. doi:10.1093/bja/aeh072

7. Harke H, Gretenkort P, Ladleif HU, Rahman S. Spinal cord stimulation in sympathetically maintained complex regional pain syndrome type I with severe disability. A prospective clinical study. Eur J Pain. 2005;9(4):363-373. doi:10.1016/j.ejpain.2004.09.003 
8. Kemler MA, de Vet HC, Barendse GA, van den Wildenberg FA, van Kleef M. Effect of spinal cord stimulation for chronic complex regional pain syndrome Type I: five-year final follow-up of patients in a randomized controlled trial. J Neurosurg. 2008;108(2):292-298. doi: $10.3171 / \mathrm{JNS} / 2008 / 108 / 2 / 0292$

9. Deer TR, Levy RM, Kramer J, et al. Dorsal root ganglion stimulation yielded higher treatment success rate for complex regional pain syndrome and causalgia at 3 and 12 months: a randomized comparative trial. Pain. 2017;158(4):669-681. doi:10.1097/j.pain.00000000 00000814

10. Huygen F, Liem L, Cusack W, Kramer J. Stimulation of the L2-L3 Dorsal Root Ganglia induces effective pain relief in the low back. Pain Pract. 2018;18(2):205-213. doi:10.1111/papr.12591

11. Amann W, Berg P, Gersbach P, et al. Spinal cord stimulation in the treatment of non-reconstructable stable critical leg ischaemia: results of the European Peripheral Vascular Disease Outcome Study (SCS-EPOS). Eur J Vasc Endovasc Surg. 2003;26(3):280-286. doi:10.1053/ejvs.2002.1876

12. Kumar A, Felderhof C, Eljamel MS. Spinal cord stimulation for the treatment of refractory unilateral limb pain syndromes. Stereotact Funct Neurosurg. 2003;81(1-4):70-74. doi:10.1159/000075107

13. Pope JE, Deer TR, Kramer J. A systematic review: current and future directions of dorsal root ganglion therapeutics to treat chronic pain. Pain Med. 2013;14(10):1477-1496. doi:10.1111/pme.12171

14. Eldabe S, Burger K, Moser H, et al. Dorsal Root Ganglion (DRG) Stimulation in the Treatment of Phantom Limb Pain (PLP). Neuromodulation. 2015;18(7):610-616; discussion 616-617. doi:10. 1111/ner. 12338

15. McAuley J, van Groningen R, Green C. Spinal cord stimulation for intractable pain following limb amputation. Neuromodulation. 2013;16(6):530-536; discussion 536. doi:10.1111/j.1525-1403.20 12.00513.x

16. Harke H, Gretenkort P, Ladleif HU, Koester P, Rahman S. Spinal cord stimulation in postherpetic neuralgia and in acute herpes zoster pain. Anesth Analg. 2002;94(3):694-700; table of contents. doi:10.1097/00000539-200203000-00040

17. Roe M, Sehgal A. Pharmacology in the management of chronic pain. Anaesth Intensive Care Med. 2016;17(11):548-551. doi:10.1016/j. mpaic.2016.08.010
18. Taylor RS. Spinal cord stimulation in complex regional pain syndrome and refractory neuropathic back and leg pain/failed back surgery syndrome: results of a systematic review and meta-analysis. J Pain Symptom Manage. 2006;31(4 Suppl):S13-S19. doi:10.1016/j. jpainsymman.2005.12.010

19. Kumar K, Malik S, Demeria D. Treatment of chronic pain with spinal cord stimulation versus alternative therapies: cost-effectiveness analysis. Neurosurgery. 2002;51(1):106-115; discussion 115-106. doi:10.1097/00006123-200207000-00016

20. Deer TR, Sayed D, Pope JE, et al. Emergence from the COVID-19 pandemic and the care of chronic pain: guidance for the interventionalist. Anesth Analg. 2020;131(2):387-394. doi:10.1213/ ANE.0000000000005000

21. Telematics WGCoH. A health telematics policy in support of WHO's Health-for-all strategy for global health development: report of the WHO group consultation on health telematics, 11-16 December, Geneva, 1997. Geneva, Switzerland; 1997.

22. Kichloo A, Albosta M, Dettloff K, et al. Telemedicine, the current COVID-19 pandemic and the future: a narrative review and perspectives moving forward in the USA. Fam Med Community Health. 2020;8(3):e000530. doi:10.1136/fmch-2020-000530

23. Ekeland AG, Bowes A, Flottorp S. Effectiveness of telemedicine: a systematic review of reviews. Int $J$ Med Inform. 2010;79 (11):736-771. doi:10.1016/j.ijmedinf.2010.08.006

24. Rojas SV, Gagnon MP. A systematic review of the key indicators for assessing telehomecare cost-effectiveness. Telemed E Health. 2008;14(9):896-904.

25. Han Y, Lu Y, Wang D, et al. The use of remote programming for spinal cord stimulation for patients with chronic pain during the COVID-19 outbreak in China. Neuromodulation. 2021;24(3):441-447.

26. Rudd RA, Seth P, David F, Scholl L. Increases in drug and opioidinvolved overdose deaths - United States, 2010-2015. MMWR Morb Mortal Wkly Rep. 2016;65(50-51):1445-1452. doi:10.15585/mmwr. $\mathrm{mm} 655051 \mathrm{e} 1$

27. Mendez I, Song M, Chiasson P, Bustamante L. Point-of-care programming for neuromodulation: a feasibility study using remote presence. Neurosurgery. 2012;72(1):99-108. doi:10.1227/NEU.0b0 $13 \mathrm{e} 318276 \mathrm{~b} 5 \mathrm{~b} 2$
Journal of Pain Research

\section{Publish your work in this journal}

The Journal of Pain Research is an international, peer reviewed, open access, online journal that welcomes laboratory and clinical findings in the fields of pain research and the prevention and management of pain. Original research, reviews, symposium reports, hypothesis formation and commentaries are all considered for publication. The manuscript

Submit your manuscript here: https://www.dovepress.com/journal-of-pain-research-journa management system is completely online and includes a very quick and fair peer-review system, which is all easy to use. Visit http:// www.dovepress.com/testimonials.php to read real quotes from published authors. 